\title{
IV
}

\section{REPORT ON THE FLORA OF ST. ANDREWS, N.B.}

\author{
BY PROFESSOR JAMES' FOWLER, LL. D., QUEEN'S UNIVERSITY, \\ KINGSTON.
}

\section{INTRODUCTORY NOTES.}

On June 9, 1900, the writer arrived at the Biological Laboratory, at St. Andrews, and devoted his time till August 18, to the study of the flora in the neighbourhood, and to the collection of herharium specimens. The special object of his visit was to collect and study the marine alge that might be found in that part of the Bay of Fundy. At the time of his arrival the retreating tide had left the rugged shore bare for a considerable distance, and the rocks, covered with a dense growth of rock-weed (Fucus) presented an attractive fifld for exploration. After spending a couple of days among the slippery rocks and mud, he discovered that very few species of algæ could be secured, and only those of the most hardy species. The rugged character of the shores, formed by the waves and tides from the red sandstone in some localities, and from volcanic rock in others, renders it impossible to travel along the beach any considerable distance in search of specimens. The aid of a boat is indispensable to the collector who wishes to extend his resear hes beyond the immediate neighbourhood of the station; but unfortunately the writer was precluded from more extended investigations. Disappointed at the small number of species where the prospects seemed so bright, he endeavoured to discover the reasons of their paucity, and is of the opinion that the following facts explain the phenomenon:-

1. The great tides of the Bay of Fundy produce currents which sweep away all plants not firmly anchored to the rocks. The fucaceæ, possessed of tough and flexible stems, and attached to the rocks by holdfasts that cannot be separated from them by any force tugging at the stems and branches, are naturally adapted to resist the action of waves and currents, while other more delicate species arre swept away and carried out to sea or thrown up on the rocky shores.

2. At low water, a large extent of shore is left bare, and the algr attached to the rocks are exposed for several hours every day to the warm winds and drying power of the summer sun. All plants unable to endure this ordeal must give place to the hardier species. The delicate forms that inhabit the pools or marshy shores are consequent!y unknown.

3. The great rise and fall of the tides stir up the waters of the bay to a great depth and as no broad areas of sand are exposed to the sun's rays to absorb heat and impart it to the waters that cover them at the return of the tide, these waters are always cold. Hence only algæ capable of flourishing in the cold waters are adapted to these rugged shores.

The combination of these factors constitutes an environment which is fatal to all but the most hardy species of littoral algæ. All delicate forms must betake themselves to retired creeks and sheltered inlets where many of them may doubtless be found ; but they can only be reached by the collector who is fortunate enough to enjoy the advantage of appropriate transit by. water.

Having failed, owing to the causes mentioned above, and the lack of necessary facilities for identifying species, to secure the number of marine plants anticipated, the 
collector immediately turned to the streets and fields of the town and its neighbourhood: which promised a more abundant harvest. During the early half of the century St. Andrews was distinguished for its great commercial activity, especially in its export of lumber. The long line of wharfs and the numerous warehouses, now falling into ruins, along the front of the town, are monuments of a prosperity which has now completely passed away with the destruction of the forests upon which it depended. Some of the streets as well as the wharfs are now almost deserted, and furnish favourable conditions. for the growth and propagation of the foreign weeds and plants imported in earlier days. Many gardens and fields have been abandoned by their owners and are now rich collecting grounds for the botanist. Plants that once ornamented the grounds of wealthy merchants or prosperous farmers, have spread to the roadside and fields, or abound on the sidewalks along the deserted streets. A large area near the town, which once constituted the town park, with its winding paths, its artificial lake and its pleasant flower beds and grass plots, is now a perfect paradise for the botanist.

The w riter can recall no locality he has ever visited where such a large number of foreign plants can be found in such a limited area, At the time of his arrival the early blooming plants had shed their flowers. The forest trees and native shrubs had passed the flowering season-had assumed their summer appearance and were now ripening their fruits. The winds were scattering the seeds of the poplars and willows over the neighbourhood where they grew. But though the spring flowers had disappeared the streets and fields were gay with the blossoms of foreign plants. Every rising sun was welcomed with a fresh display of floral beauty.

For several weeks Ranunculus repens, L., whether native or introduced, displayed its large yellow flowers abundantly in the ditches along the streets and in the damp grounds; and the common Buttercup (Ranunculus acris, $\mathbf{L}$.) adorned the higher grounds. The Wild Mustard (Brassica arvensis, L.) has pushed its way successfully out into the open country and many fields were brilliant with its yellow petals. Two other species (Brassica nigra, Koch. and B. campestris, L.) occupied more limited areas, but added to the general display. Another member of the Cruciferous family (Lepidium ruderals, L.) found a congenial home on the decaying wharfs. Among the introduced forms, which have secured a permanent home for themselves, few have become more conspicuous than the yellow clover (Trifolium procumbens, L.) It has spread over roads and railroad tracks in different localities to the almost total exclusion of the other species. It must, however, yield the palm to the Carroway (Carum carui, L.) which has not only invaded the town but has overrun the entire country for miles around. If the seeds were collected a sufficient quantity would be obtained to supply the demands of the province, perhaps of the Dominion. Of thirty.two species of Compositæ collected, twenty have been introduced from foreign lands. The less frequented streets were brilliant during the month of June with Dandelions of which two species occur (Taraxacum taraxacum, Karst, and T'. eryth,ospermum, Andrz). The latter must be rare as the writer has never noticed it elsewhere. One of the most interesting members of this family is the Hieracium aurantiacum, L., which is exceedingly abundant near the laboratory, but has not spread into the fields. Leontodon autumnalis, $\mathrm{I}_{\text {., meets the }}$ eye erery where, and Tragopogon pratensis is common in deserted gardens and fields. The Blue-bell family (Campanulacex) is represented by large numbers of Campanula rapunculoides, L., whose long racemes of blue flowers with corollas an inch in length are very conspicuous on the sidewalks and along the garden fences.

Of the native plants in the immediate neighbourhood of the laboratory in the months of June and July the following species are most likely to attract the attention of the visitor from the west :-

Viola primulaefolia, L

Viola lanceolata, L.

Potentilla tridentata, Ait.

Potentilla anserina, $\mathbf{L}$.

Rosa humilis lucida, Ehrh.

Drosera rotundifolia, $\mathbf{L}$.

Aster tardiflorus, L.

Antennaria neodioica, Greene.
Rhodora Canodensis, L.

Euphrasia Americana.

Rhinanthus Crista-Galli, L.

Carex Goodenovii, J. Gay.

Carex maritima, Muller.

Poo flava, $L$.

Festuca ovina duriuscula, L.

Botrychium simplex, Hitehcock. 


\section{SESSIONAL PAPER No. 22a}

The following probably mark the sites of former gardens:-

Tilia Europœa, L.

Geranium pratense, $\mathbf{L}$.

Asculus hippocastanum, L.

Acer platanoides, $\mathrm{L}$.

Acer pseudo-platanus, $\mathbf{L}$.

Robinia psendacacia, $\mathbf{L}$.

Caragana arborescens, Lam.

Spiraea sorbifolia, L.

Spiraea ulmaria, L

Crataegus oxyacantha, L.

Philadelphus coronarius, $\mathbf{L}$
Sedum acre, $\mathbf{L}$

Diervilla florida, Sieb. \& Zucc.

Centaurea nigra, $\mathbf{L}$.

Syringa vulgaris, $\mathbf{L}$.

Leptandra Virginica, Nutt.

Euphorbia Cyparissias, L.

Ulmus campestris, $\mathbf{L}$.

Larix Europaea, D.C.

Hemerocallis fulva, $\mathrm{L}$.

Lysimachia nummularia, L.

BOTANICAL LIST.

List of plants collected at St. Andrews, N.B., between June 9 and August 18, 1900.

NoTe-The Nomenclature follows that of Brown \& Britton, Illustrated Flora.

Order I. Ranunculaceg.

Genera. Spec.

1 Thalictrum polygamum, Muhl.

2 Ranunculus repens, $\mathbf{L}$.
Genera. Spec.

$\begin{array}{lcl}3 & 4 & \text { Oxygraphis Cymbalaria, Prantl. } \\ 4 & 5 & \text { Coptis trifolia, Salisb. } \\ 5 & 6 & \text { Actaea rubra, Willd. }\end{array}$

ORDER II. NYMPHAEACEE.

$6 \quad 7$ Castalia odorata, Woodv.

7

8 Nymphaea advena, Soland.

\section{Order III. Crucifere.}

8
9
10

9 Barbarea barbarea, MacM.

10 Erysimum cheiranthoides, L.

11 Brassica arvensis, $\mathrm{L}$.

12 Brassica nigra, Ksch.

13 Brassica campestris, L.

$\begin{array}{ccl}11 & 14 & \text { Bursa bursa-pastoris, Britton. } \\ 12 & 15 & \text { Lepidium ruderale, L. } \\ 13 & 16 & \text { Cakile edentula, Hook. } \\ 14 & 17 & \text { Raphanus raphanistrum, L. }\end{array}$

Order IV. Violacea.

18 Viola obliqua, Hill.

19 Viola blanda, Willd.

20 Viola primulaefolia, L.

21 Viola lanceolata, $\mathrm{L}$.

Order $V$. Caryophyllacee.

22 Moehringia lateriflora, L.

Alsine media, $L$

Alsine longifolya, Britton.

Alsine graminea, Britton.

Alsine bumifusa, Britton.

1827 Cerastium vulgatum, L.

1928 Sagina procumbens, $\mathrm{L}$.

2029 Tissa rubra, Britton.

30 Tissa Canadensis, Britton.

2131 Spergula arvensis, L.

Order Vi. Hypericaces.

34 Hypericum Canadense, L.

Order VII. Tiliacea. 


\section{Order VIII. Geranicade.}

Genera. Species.

$\begin{array}{lll}24 & 37 & \text { Geranium pratense, } \mathbf{L} . \\ 25 & 38 & \text { Oxalis acetosella, } \mathbf{L} \text {. }\end{array}$
Genera. Species.

36 - Oxalis stricta, L.

2640 Impatiens biflora, Walt.

Order IX. Illicinee.

27

41 Tlex verticillata, Gray.

\section{Order X. Sapindacede.}

2842 Asculus Hippocastanum, L.

2943 Acer spicatum, Lam.
44 Acer platanoides, L.

45 Acer pseudo-platanus, $L$.

Order XI. Leguminosa.

30

46 Trifolium pratense, $\mathrm{L}$.

47 Trifolium repens, $\mathrm{L}$.

48 Trifolium procumbens, $\mathrm{L}$.

31

Melilotus officinalis, Willd.

50 Melilotus alba, Lam.

32

51 Medicago lupulina, L.
$33 \quad 52$ Robinia pseudacacia, $\mathrm{L}$.

$34 \quad 53$ Vicia cracca, $\mathbf{L}$.

$35 \quad 54$ Lathyrus maritimus, Bigel.

55 Lathyrus palustris, L.

$36 \quad 56$ Caragana arborescens, Lam.

\section{Order XII. Rosaces.}

57 Prunus virginiana, $L$.

58 Spiræa salicifolia, $L$.

59 Spiræa tomentosa, $\mathbf{L}$.

60 Spiræa sorbifolia, $\mathbf{L}$.

61 Spiræa ulmaria, L.

62 Rubus Americanus, Britton.

63 Rubus strigosus, Miche.

64 Rubus villosus frondosus, Bigel.

$40 \quad 65$ Geum strictum, Ait.

$41 \quad 66$ Fragaria virginiana, Mill.

4267 Potentilla norvegica, $L$.

$\begin{array}{lll} & 68 & \text { Potentilla argentea, I. } \\ & 69 & \text { Potentilla tridentata, Ait. } \\ & 70 & \text { Potentilla anserina, L. } \\ & 71 & \text { Potentilla Canadensis, L. } \\ 43 & 72 & \text { Comarum palustre, L. } \\ 44 & 73 & \text { Rosa humilis lucida, Best. } \\ 45 & 74 & \text { Cratæous oxyacantha, L. } \\ 46 & 75 & \text { Aronia nigra, Britton. } \\ 47 & 76 & \text { Sorbus Americana, Marsh. } \\ & 77 & \text { Sorbus sambucifolia, Roem. }\end{array}$

Order XIII. SAXIFragace

78 Philadelphus coronarius, L.

$49 \quad 79$ Ribes oxyacanthoides, $\mathrm{L}$.

Order XIV.-Crassulacez.

80 Sedum acre, I.

Order XV.-Droserace.t.

81 Drosera rotundifolia, L.

\section{Order XVI.-HaLoragea.}

82 Callitriche palustris, L.

\section{Order XVII.-Onagracez.}

86 Epilobium adenocaulon, Haussk.

87 Onagra biennis, Scop.

88 Kneiffia pumila, Spach.

89 Circæa alpina, L. 
SESSIONAL PAPER No: 22a

ORDER XVIII.-UMBIFELLIFERA.

Genera. Species.

58 ... 90 Carum carui, L.

59 -. 91 Cicuta bulbifera, L

Genera. Species.

6092 Hydrocotyle Americana, L.

6193 Ligusticum Scoticum, L.

Order XIX.-Araliace.e.

62

94 Aralia hispida, Vent.

95 Aralia nudicaulis, $\mathbf{L}$.

\section{Order XX.-Cornaces.}

96 Cornus Canadensis, $\mathbf{L}$.

\section{Order XXI.-Caprifoliaces}

$64 \quad 97$ Viburnum cassinoides, $L$.

6598 Linnæa borealis, L.

6699 Diervilla Diervilla, MacM.

100 Diervilla florida, Sieb. \& Zucc.

\section{Order XXII.-Rubiacef.}

101 Houstonia coerulea, L.

\section{Order XXIII.-Composite.}

$\begin{array}{lll}68 & 102 & \text { Eupatorium perfoliatum, L. } \\ 69 & 103 & \text { Solidago puberula, Nutt. } \\ & 104 & \text { Solidago juncea, Ait. } \\ & 105 & \text { Solidago rugosa, Mill. } \\ & 106 & \text { Solidago Oanadensis, L. } \\ 70 & 107 & \text { Eutbamia giaminifolia, Nutt. } \\ 71 & 108 & \text { Aster tardifiorus, L. } \\ & 109 & \text { Aster lateriflorus, Britton. } \\ 80 & 118 & \text { Achilea millefolium, L. } \\ 81 & 119 & \text { Chrysanthemum leucanthemum, L. } \\ 82 & 120 & \text { Artemisia vulgaris, L. } \\ 83 & 121 & \text { Senecio vulgaris, L. } \\ 84 & 122 & \text { Antennaria neodioica, Greene. } \\ 85 & 123 & \text { Arctium minus, Schk. } \\ 86 & 124 & \text { Carduus arvensis, Robs. } \\ 87 & 125 & \text { Centaurea nigra, L. }\end{array}$

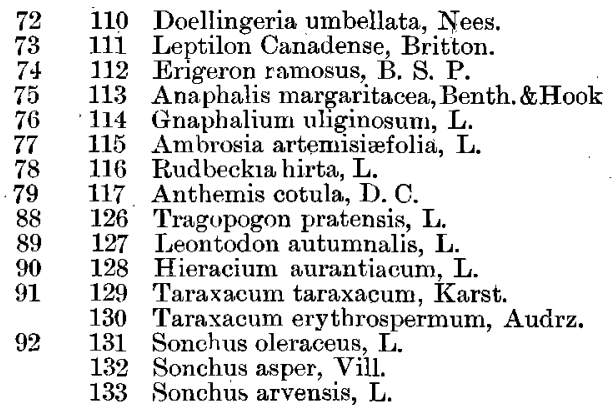

Order XXIV. Lobeliaces.

134 Lobelia inflata, I.

138 Lobelia Dortmanna, L.

\section{Order XXV. Campanulaces.}

Order XXVI. Ericaces.

138 Vaccinium Pennsylvanicum, Lam.

$98 \quad 143$ Rhodora Canadensis, I

139 Vaccinium Canadense, Richards.

140 . Vaccinium vitis-id:za, $L$.

96141 Oxycoccus macrocarpus, Pers

$97 \quad 142$ Kalmia angustifolia, $L$.

\section{Ledum Groenlandicum, Fder. \\ 100145 Pyrola elliptica, Nutt.}

$101 \quad 146$ Monotropa uniflora, L.

\section{Order XXVII. Plumbaginaceze.}

147 Limonium Carolinianum, Britton.

\section{Urder XXViII. Primulace a.}

$103 \quad 148$ Trientalis Americana, Pursh.

104149 Lysimachia terrestris, B.S.P.
150 Lysimachia nummularia, L.

105151 Glaux maritima, L:

\section{Order XXIX. Oleacea.}


Order XXX. Gentianacea.

Genera. Species.

108154 Menyanthes trifoliata, L.

Genera. Species.

\section{Order XXXI. Boraginacere.}

109155 Myosotis arvensis, Hoff $m$.

110156 Lappula Lappula, Karst.

$111 \quad 157$ Pneumaria maritima, Hill.

Order XXXII. Convolvulacez.

158 Convolvulus sepium, L.

\section{Order XXXIIT. Scrophulariacea.}

113

114

115

159 Linaria linaria, Karst.
160 Chelone glabra, L.
161 Leptandra Virginica, Nutt.

Order XXXIV. Labiate.

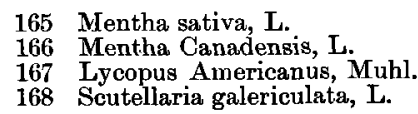

122169 Prunella vulgaris, L.

123170 Galeopsis tetrabit, $\mathrm{I}$

$124 \quad 171$ Stachys palustris, L.

Order XXXV. Plantagenacez.

\section{Order XXXVI. Chenopodiaces.}

175 A triplex hastata, $\mathrm{L}$

176 Salicornia herbacea, $\mathrm{L}$.

129177 Dondia Americana, Britton.

\section{Order XXXVII. Polygonaces.}

178 Rumex Brittanica, L.

179 Rumex acetosella, L.

131180 Polygonum aviculare, $\mathbf{L}$

181 Polygonum erectum, $\mathrm{L}$.

182 Polygonum Persicaria, $\mathrm{L}$

183 Polygonum sagittatum, $\mathrm{I}$.

184 Polygonum convolvulus, $\mathrm{L}$

\section{Order XXX ViII. Euphorbiacea.}

185 Euphorbia Cyparissias, L.

\section{Order XXXIX. Urticacea.}

186 Ulmus campestris, L.

Order XL. Myricaces.

187 Myrica gaie, L.

Order XLI. Cupulifere.

188 Betula lutea, L.

189 Betula populifolia, Ait.

136199 Alnus alnobetula, Koch

191 Alnus incana, Willd.

Order XLII. Salicacere.

192 Salix lncida, Muhl.

194 Salix balsamifera, Barratt.

193 Salix Bebbiana, Sarg.

\section{Order XLIII. Conhfere.}

195 Larix Jaricina, Koch.

196 Larix Europea, DC.

197 Thuya occidentalis, $\mathbf{L}$.
140198 Juniperus nana, Willd.

199 Juniperus Sabina, L. 
SESSIONAL PAPER No. 22a

Order XLIV. Orchidace.t.

Genera. Species

141200 Achroanthes unifolia, Raf.

142201 Leptorchis Loeselii, MacM.

143202 Corallorhiza multiflora, Nutt.

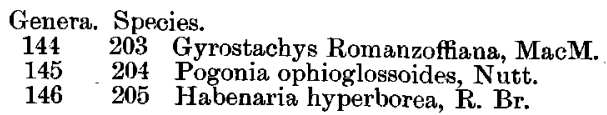

Order XLV. Iridace

117206 1ris versicolor, L.

148207 Sisyrinchium angustifolium, Mill.

Order XlVi. LiLiaces.

149208 Hemerocallis fulva, L.

150209 Vagnea stellata, Morong.

151210 Unifolium Canadense, Greene.

\section{Order XLVII. Juncaces.}

212 Juncus effusus, L.

213 Junous Balticy, Willd.

214 Juncus Gerard, Loisel.

215 Juncus bufonius, $\mathbf{L}$.

216 Juncus articulatus, $I$.

217 Juncus Canadensis brevicaudatus, Engelm.

154218 Juncoides campestre, Kuntze.

Order Xliviti. Typhacez.

219 Typha latifolia, L.

\section{Order XliX. Alismaces.}

220 Sagittaria latifolia, Willd,

\section{Order L. Naiadaces.}

221 Triglochin maritima, L.

222 Potamogeton Nuttallii, Cham. \& Sch. $159 \quad 223$ Zostera marina, $\mathbf{L}$.

\section{Order LI. Cyperace}

224 Eleocharis ovata, $\mathrm{B}, \mathrm{Br}$

225 Eleocharis palustris glaucescens, Gray.

226 Eleochar is tenuis, Schutes.

227 Scirpus microcarpus, Presl.

228 Scirpus atrovirens, Muht.

229 Scirpus fluviatilis, Gray.

230 Scirpus cyperinus, L.

231 Scirpus Americanus, Pers.

232 Eriophorum Virginicurr, $\mathbf{L}$.

233 Carex arctata, Boot.

234 Carex aurea, Nutt.

235 Carex brunnescens gracilior, Britton.

236 Carex canescens, $\mathrm{L}$.

237 Carex crinita, Lam

238 Carex flava, $\mathrm{L}$.
239 Carex Goodenovii, J. Gay.

240 Carex intumescens, Rudge.

241 Carex lurida, Wahl

242 Carex maritima, Muller.

243 Carex Novæ-Angliæ, Schwein.

244 Carex pallescens, I.

245 Carex pedicellata, Britton.

246 Carex scoparia, Schk.

247 Carex sterilis, Willr.

248 Carex sterilis cephalantha, Bailey.

249 Carex stipata, Muhl.

250 Carex tenera, Dewey.

251 Carex tenuis, Rudge

252 Carex retrorsa, Schwein.

253 Carex viridula, Michx.

\section{ORDER LTI.-GRAMINEe.}

254 Spartina cynosuroides, Willd.

255 Spartina patens, Muhl.

256 Spartina stricta maritima, Scrib.

257. Panicum implicatum, Scrib.

259 Anthoxanthum odoratum, L.

260 Phleum pratense, $\mathrm{I}$.

261 Alopecurus geniculatus, $\mathbf{L}$.

262 Agrostis alba, L.

263 Agrostis hyemalis, B.S.P.

264 Danthonia spicata, Beauv.

265 Poa compressa, $\mathrm{L}$.

266 Poa flava, L.

$\begin{array}{lll} & 267 & \text { Poa pratensis, L. } \\ & 268 & \text { Poa trivialis, L. } \\ 172 & 269 & \text { Panicularia Canadensis, Kuntze. } \\ & 270 & \text { Panicularia nervata, Kuntze. } \\ & 271 & \text { Panicularia Americana, MacM. } \\ 173 & 272 & \text { Puccinella maritima, Parl. } \\ 174 & 273 & \text { Dactylis glomerata, L. } \\ 175 & 274 & \text { Festuca ovina duriuscula, L. } \\ & 275 & \text { Festuca elatior, L. } \\ 176 & 276 & \text { Agropyron repens, L. } \\ 177 & 277 & \text { Hordeum jubatum L. } \\ 178 & 278 & \text { Elymus arenarius; } \mathbf{L} .\end{array}$

267 Poa pratensis, L.

Poa trivialis, L.

70 Panicularia nervata, Kuntze.

Panicularia Americana MacM.

3 Dactylis glomerata. $\mathbf{L}$.

6 Agropyron repens,

278 Elymus arenarius, $\mathbf{I}$.

\section{Order LiII. - Equisetacea.}


Order LIV.-Filices.

\begin{tabular}{|c|c|c|c|}
\hline Genera. & Specie & & Genera. \\
\hline 180 & 281 & Polypodium vulgare, L. & \\
\hline 181 & 282 & Pteris aquilina, $\mathrm{L}$. & \\
\hline 182 & 283 & Asplenium filix-foemina, Bernh. & 185 \\
\hline $\begin{array}{l}102 \\
183\end{array}$ & 284 & Phegopteris Phegopteris, Underw. & 186 \\
\hline 184 & $\begin{array}{l}286 \\
287\end{array}$ & $\begin{array}{l}\text { Dryopteris spinulosa intermedia, Und } \\
\text { Dryopteris spinulosa dilatata, Underw }\end{array}$ & 188 \\
\hline
\end{tabular}

Species.

288 Dryopteris cristata, Gray.

289 Dryopteris acrostichoides, Sw.

290 Onoclea sensibilis, $\mathbf{L}$.

291 Woodsia ilvensis, $R$. Br.

292 Dicksonia punctilobula, Gray.

293 Osmunda Claytoniana, L.

294 Osinunda cinnamomea, $\mathbf{L}$.

\section{Order LV. - Ophioglossacez.}

\section{Order LVI.-Lycopodiacea.}

297 Lycopodium lucidulum, Michx.

299 Lycopodium complanatum, L.

298 Lycepoditim obscurum, L.

\section{MUSCI.}

\section{Order LVII.-SPhagnaceA.}

\section{Order LVIII.-Bryacee.}

302 Leucobryum glaucum, L.

302 Ceratodon purpureus, $L$.

303 Ulota crispa, Brid.

304 Ulota crispula, Brid.

305 Ulota Ludwigii, Brid.

195

306 Polytrichum commune, L.
307 Polytrichum juniperinum, Willd

308 Webera nutans (Schreb.) Hedw.

309 Pylaisia Schimperi, Card.

310 Aulacomnium palustre. Schwaegr.

311 Hypnum uncinatum, Hedw.

\section{Order LIX.—JungermanNiacez.}

312 Ptilidium ciliare, Nees.

\section{LICHENES.}

313 Alectoria jubata, $\mathbf{L}$.

314 Usnea barbata, L.

315 Theloschistes parietinus, $L$.

316 Sticta pulmoraria, L.

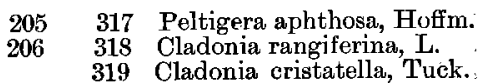

ALG正.

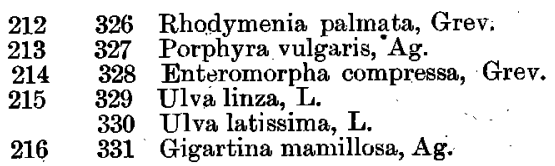

319 Cladonia cristatella, Tuck.
320 Fucus vesiculosus, $\mathrm{L}$.

321 Fucus nodosus, I.

322 Laminaria longicruris, De la Pyl.

323 Chordaria flagelliformis, Ag.

324 Polysiphonia fastigiata, Grev.

325 Corallina officinalis, $\mathbf{L}$.
Several specimens of Algæ collected in addition to the foregoing have not yet been determined: 